\title{
Fe-Deficiency in H-Deficient Post-AGB Stars Due to n-Capture Nucleosynthesis
}

\author{
Falk Herwig \\ University of Victoria, Victoria, BC, Canada \\ Maria Lugaro \\ IoA, University of Cambridge, Cambridge, UK \\ Klaus Werner \\ Universität Tübingen, Tübingen, Germany
}

\begin{abstract}
H-deficient post-AGB objects, e.g. PG1159 type star K116 and born-again AGB star Sakurai's object, have been reported to be significantly iron-deficient. We find that the iron deficiencies expected due to neutron-capture nucleosynthesis during either the progenitor AGB evolution and/or the neutron burst that occurs as a result of the rapid burning of protons during a post-AGB He-flash are generally in line with observations.
\end{abstract}

Recently, Miksa et al. (2001) reported that the PG1159 central star (CS) K1-16 is deficient in iron by at least a factor 10-100 (see also contributions by Werner, Gräfener et al. and Crowther at this conference and Miksa et al., 2002). PG1159 stars are likely the result of a born-again evolution associated with a thermal pulse (TP) occurring during the post-Asymptotic Giant Branch phase (postAGB). In addition, Sakurai's object, which experienced a very late TP (VLTP, see Herwig et al. (1999), shows an Fe-depletion of about 1dex and a $\mathrm{Fe} / \mathrm{Ni}$ ratio of only $\sim 3$ (Asplund et al., 1999).

The material of H-deficient post-AGB stars reflects the intershell abundance of the progenitor AGB star with some superimposed modification due to the mixing and/or nucleosynthesis processes which lead to the formation of the Hdeficiency. It has been established that the $s$-process in AGB stars is the result of recurrent neutron exposure episodes in the region between the $\mathrm{He}-$ and $\mathrm{H}$ burning shell. Neutron exposures lead to a significant depletion of iron in this layer. Therefore the final AGB intershell abundances have to be taken as initial conditions for VLTP nucleosynthesis models. These have been obtained with the AGB $s$-process code TOSP (Gallino et al., 1998), which computes the recurrent neutron exposures and mixing events over many TPs using thermodynamic data from full stellar evolution calculations. As shown in Fig. 1 (left panel) Fe is noticeably depleted in the intershell of evolved AGB stars and the results depend significantly on the stellar models on which the $s$-process calculation is based. We have created a number of one-zone nucleosynthesis models for conditions encountered during a VLTP (Herwig, 2001) with a fully implicit iterative nuclear 

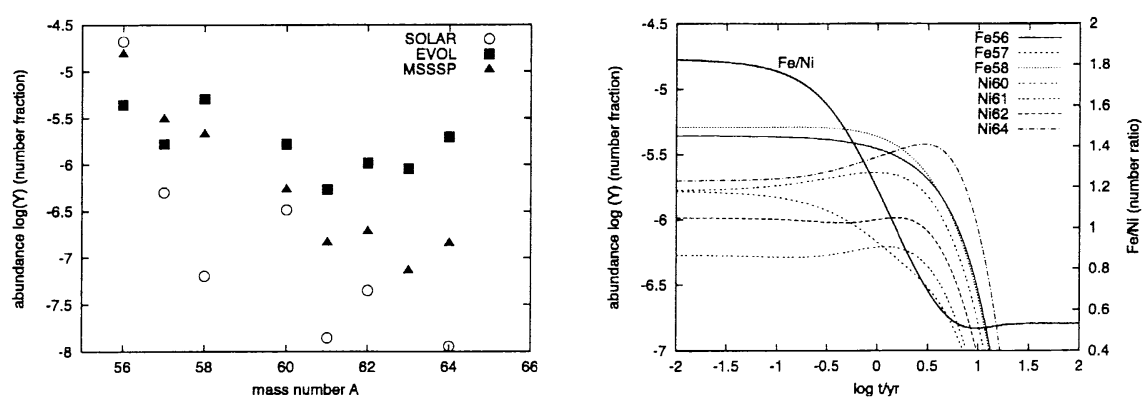

Figure 1. Left: $\mathrm{Fe}(\mathrm{A}=56-58)$ and $\mathrm{Ni}(\mathrm{A}=60-64)$ isotopic abundances in the intershell at the end of the AGB evolution $\left(3 \mathrm{M}_{\odot}, \mathrm{Z}=0.02\right)$ as computed with the TOSP post-processing code, using either the stellar evolution sequences of the EVOL code with overshoot or the MSSSP code without overshoot as input (for more details see Lugaro \& Herwig, 2001) and for comparison the SOLAR abundances. Right: Fe and $\mathrm{Ni}$ isotopes from a one-zone model which simulates a VLTP $\left(T_{8}=1.25\right.$, $\left.X_{\text {ini }}(\mathrm{H})=0.01\right)$. Initial abundances are shown left, case EVOL.

network code. The characteristic ingestion of hydrogen into the He-shell during a VLTP is simulated by setting the initial $\mathrm{H}$-abundance $X_{\text {ini }}$ to a non-zero value. We chose temperatures and densities as typically encountered at the bottom of the $\mathrm{H}$-ingestion convection zone. These conditions do persist only for a fraction of a year. For the specific parameters of the simulation shown in Fig. 1 (right panel) a considerable depletion of iron in addition to the Fe-depletion from the AGB phase is found. However, at this stage of modeling the exact numbers should not be taken too seriously. The one-zone models can only reveal the main processes involved and more elaborate models will be constructed.

Acknowledgments. F.H. would like to thank D.A. VandenBerg for support through his Operating Grant from the Natural Science and Engineering Research Council of Canada.

\section{References}

Asplund, M., Lambert, D. L., Kipper, T., Pollacco, D., \& Shetrone, M. D. 1999, A\&A, 343,507

Gallino, R., Arlandini, C., Busso, M., Lugaro, M., Travaglio, C., Straniero, O., Chieffi, A., \& Limongi, M. 1998, ApJ, 497, 388

Herwig, F. 2001, ApJ Lett., 554, L71

Herwig, F., Blöcker, T., Langer, N., \& Driebe, T. 1999, A\&A, 349, L5

Lugaro, M. \& Herwig, F. 2001, Nucl. Phys. A, 688, 201, astro-ph/0010012

Miksa, S., Deetjen, J., Dreizler, S., et al. 2001, in ASP Conf. Ser., Vol. 226, 12th European Workshop on White Dwarfs, ed. H. Shipman, J. Provencal J. MacDonald \& S. Goodchild, 60

Miksa, S., Deetjen, J.L., Dreizler, S., et al. 2002, A\&A submitted 